\title{
Orthodontic correction of severely rotated maxillary central incisor in a diabetic adult
}

\author{
Rajesh Gyawali, Prabhat Ranjan Pokharel, Jamal Giri, Ujwal Gautam \\ Department of Orthodontics, BPKIHS, Dharan, Nepal
}

\author{
Correspondence \\ Dr. Rajesh Gyawali \\ Department of Orthodontics, \\ BPKIHS, Dharan, Nepal \\ Email: \\ gyawalirajesh@gmail.com
}

DOI: http://dx.doi.org/10.3126/ jcmsn.v11i3.14061

\begin{abstract}
Background: Orthodontics has recently seen an increase in the number of adult population seeking treatment. Financial dependency, increasing awareness and availability of service can be the reasons behind this rise. Though, clinical myths regarding duration, effectiveness of treatment, associated systemic conditions still exist, these should be of no concern and with adequate monitoring and procedural modifications, conventional orthodontic treatment is possible. Case description: A 58 year old Type II diabetic male presented to orthodontic clinic with unesthetic gap between upper front teeth. The history revealed extraction of painful mesiodens. On examination, the patient had Class I molar, canine and incisor relationship. 21 was rotated with $5 \mathrm{~mm}$ of space between central incisors. Fixed orthodontic treatment was planned after physician consultation regarding his diabetic condition. Bondable buccal tubes instead of bands were used in first molars, 0.022 " Roth brackets were bonded on other maxillary teeth. The wire gradually progressed from 0.014 "NiTi, 0.016 "NiTi to 0.018 "SS. Lingual button was attached on the labial and lingual surface of 21 to apply couple. After the correction of rotation of 21, remaining space closure with esthetic contouring of 21 was done. Maintenance of adequate oral hygiene was reinforced throughout the treatment period. Fixed lingual retainer was bonded and pericision performed to retain the achieved result. Conclusion: Orthodontic treatment can be carried out in diabetic adults with good glycemic control to achieve esthetic results; however, measures for maintenance of adequate oral hygiene should be undertaken. Interdisciplinary approach involving restorative procedures can enhance the esthetics achieved.

Keywords:Adult Orthodontics, Diabetes, Fixed Lingual Retainer, Rotation
\end{abstract}

Citation: Gyawali R, Pokharel PR, Gir i J, Gautam U. Orthodontic correction of severely rotated maxillary central incisor in a diabetic adult. JCMS Nepal. 2015;11(3):30-34.

\section{INTRODUCTION}

Orthodontics has recently seen an increase in the number of adult population seeking treatment. ${ }^{1}$ The treatment was previously confined to children and adolescent population. Financial dependency, increasing awareness, availability of service, social acceptability to orthodontic appliances, introduction of clear aligners, lingual appliances and peer recommendation can be the reasons behind this rise. ${ }^{2,3}$ Esthetics is of major concern ahead of function and stability for such group of patients. A significant number of the population also presents as a part of a comprehensive treatment plan involving TMJ, periodontal and restorative issues. ${ }^{4}$
These adults are past the growing age as the growth of craniofacial complex has already completed. So, the treatment options are limited. Growth modification is not applicable due to lack of growth. Orthodontic camouflage and orthognathic surgery are the only available options. Physiological age changes of varying degree, occurring in bone and periodontal ligament tissues; delayed bone healing in extraction socket, dense cortical bone, increased osteoclastic activity, thinning of trabeculae results to less responsiveness to orthodontic force and increased risk of marginal bone loss. ${ }^{5}$ The quantitative and qualitative changes 
in bone and compromised periodontal support require special attention. ${ }^{6}$

Besides, orthodontists need to be aware of the chronic medical conditions they are suffering from and their potential implication on orthodontic treatment. Systemic conditions like diabetes pose added complexity. Increased risk of periodontitis in diabetic patient can speed up destruction of tooth supporting structures. ${ }^{5}$ Periodontal disease has been added as a sixth classic complication of diabetes along with microangiopathy, neuropathy, nephropathy, macrovascular diseases and delayed wound healing. ${ }^{7}$ Moreover, orthodontist should keep in mind regarding diabetic microangiopathy which may lead to pulpitis, odontalgia, percussion sensitivity and even loss of vitality. ${ }^{8}$

Though, clinical myths regarding duration, effectiveness of treatment and associated systemic conditions still exist in orthodontic practice; studies have shown that these should be of no concern with adequate monitoring and procedural modifications. ${ }^{9}$ This article presents a case of an adult patient with diabetes presenting for treatment of unesthetic gap between upper front teeth due to malalignment.

\section{CASE DESCRIPTION:}

This is a case of 58 year old male patient who presented to the orthodontic clinic with the chief complaint of the unesthetic gap between upper front teeth. There was no significant family history. The patient was non-smoker and non-alcoholic. He was a diagnosed case of diabetic (type II) under oral medication. He had mildly convex facial profile. The face was apparently symmetrical and lips were competent. On examination, he had Angle's Class I molar, canine and incisor relationship with severe mesio-palatal rotation of 21 . The rotated incisor was smaller in size with irregular incisal edge as compared to the adjacent central incisor. Mesiodens was extracted because of acute pulpitis secondary to caries. Electric pulp test in 21 showed it as vital. There was a space of $5 \mathrm{~mm}$ between two central incisors. (Fig. 1) Radiographs were taken to evaluate the alveolar bone height. (Fig. 2)

\section{Treatment options:}

Based on the findings, there were two treatment options. First, align 21 with lingual surface facing labially and contour it with restoration. Second, complete derotation of 21 with minor restorative contouring. Both treatment options were discussed with the patient, including pros and cons of both the options. Finally the second option was chosen.

\section{Treatment progress:}

Proper counseling and patient motivation was done before the fixed orthodontic treatment. The patient was referred to an Internist for evaluation of glycemic level and to a periodontist for bacterial plaque control and oral hygiene instruction. The patient was instructed to continue with his daily medication and maintain good oral hygiene with

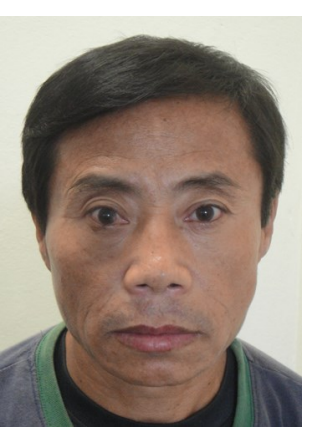

Figure 1a

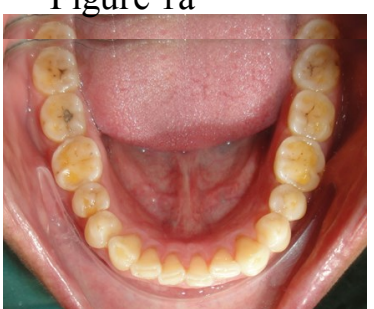

Figure 1e

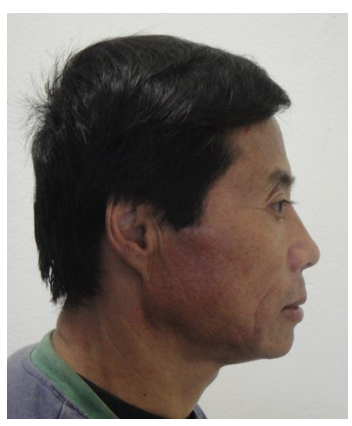

Figure $1 b$

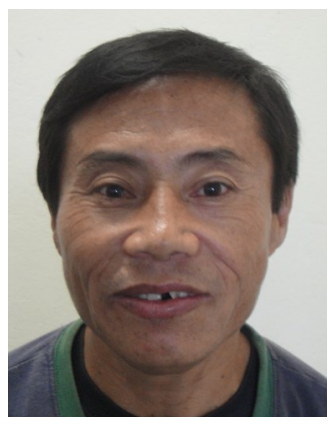

Figure 1c

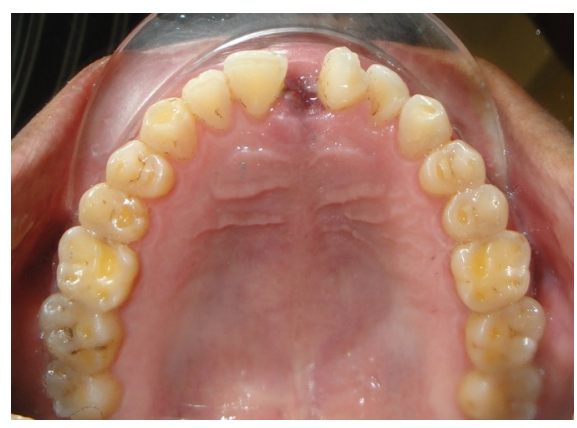

Figure 1d

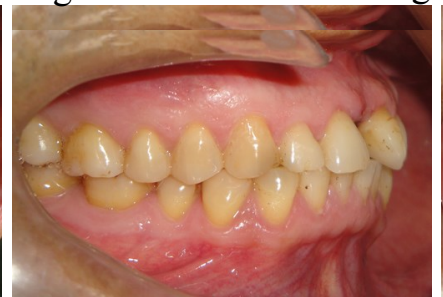

Figure 1f

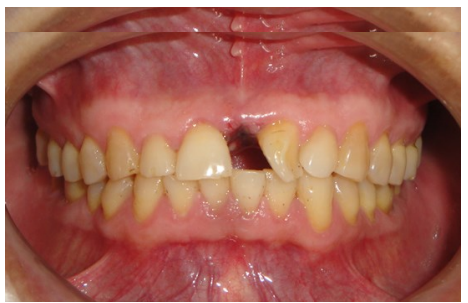

Figure $1 \mathrm{~g}$

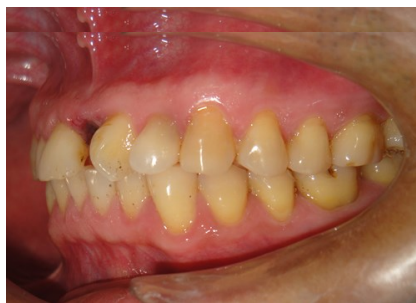

Figure 1h

Figure 1: Pretreatment extraoral and intraoral photographs 


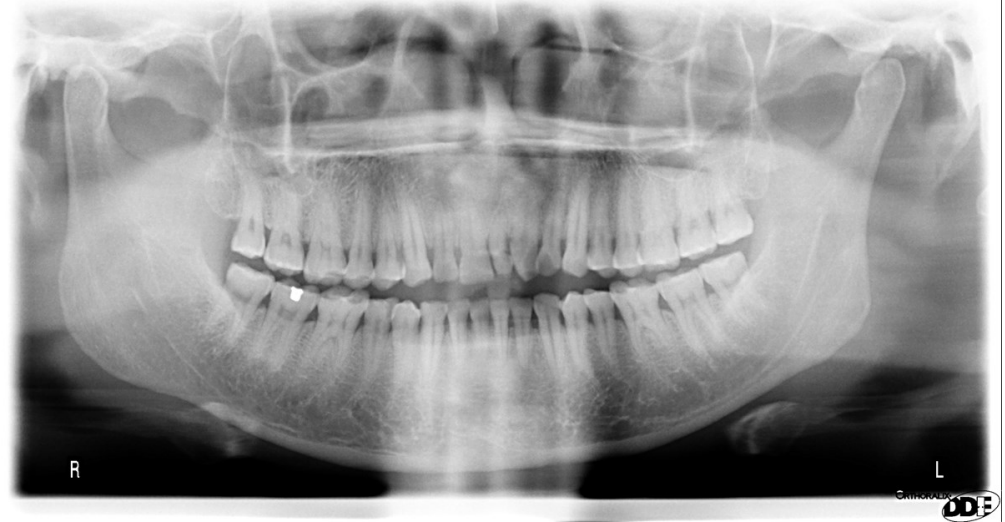

Figure 2a

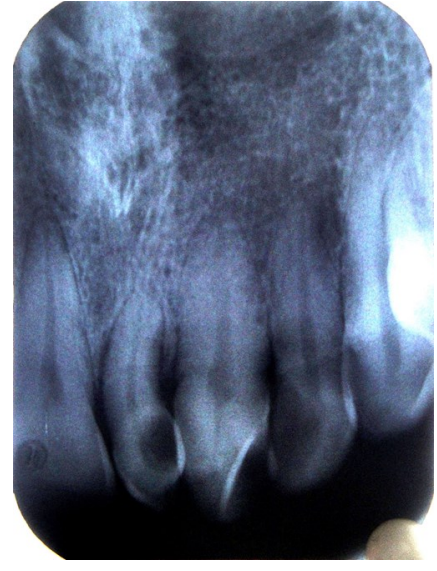

Figure $2 b$

Figure 2: Pretreatment radiographs. a- panoramic view

b- intraoral periapical radiograph of 21

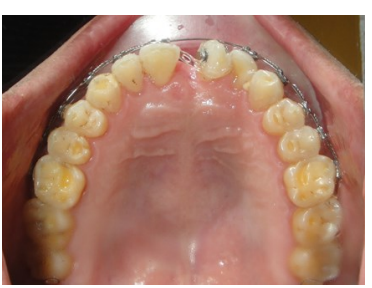

Figure 3a

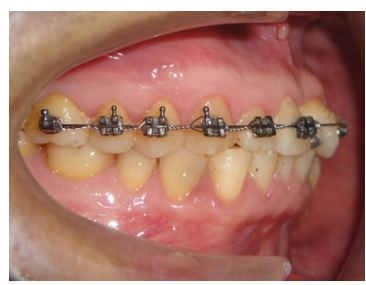

Figure $3 b$

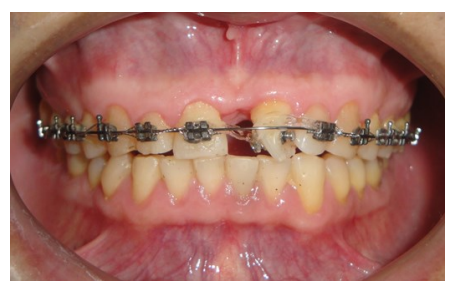

Figure 3c

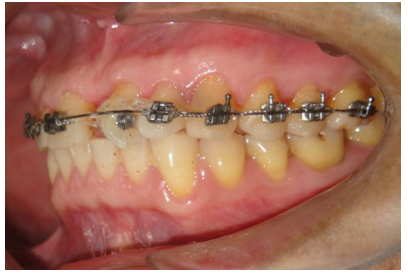

Figure 3d

Figure 3: Couple to derotate 21 in rigid 0.018 " stainless steel archwire

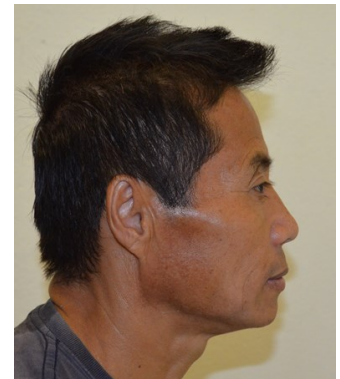

Figure $4 \mathrm{a}$

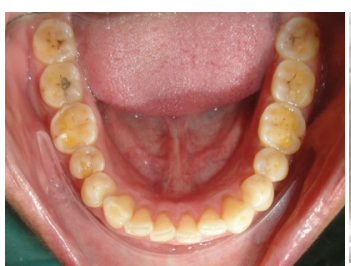

Figure 4e

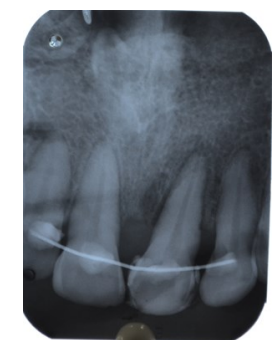

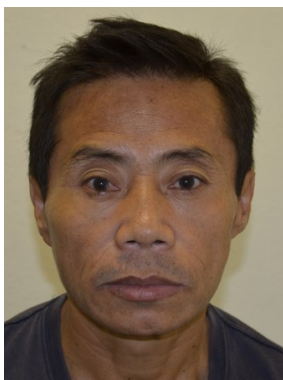

Figure $4 b$

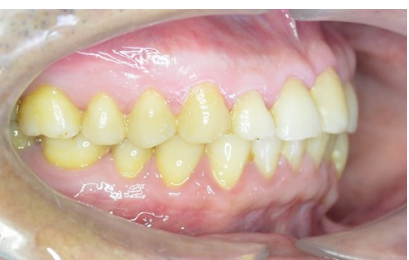

Figure 4f

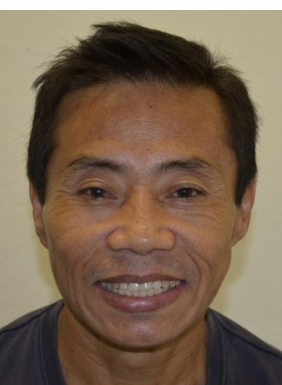

Figure $4 c$

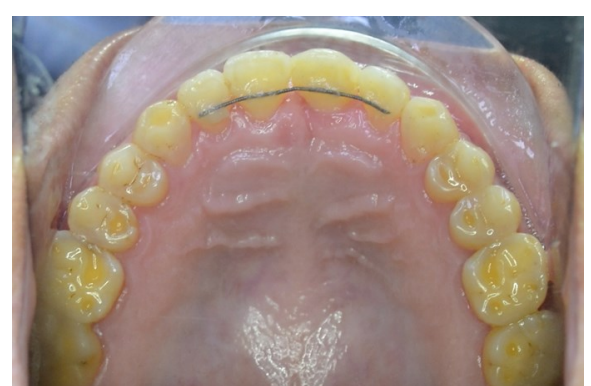

Figure 4d

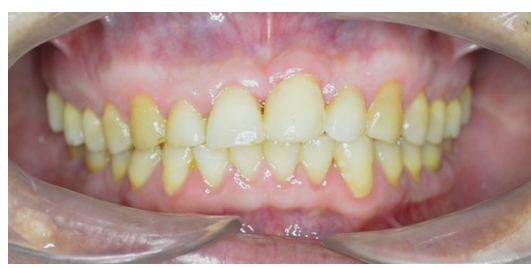

Figure 4g

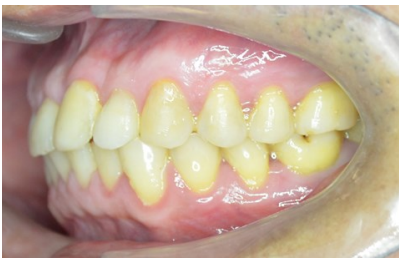

Figure $4 \mathrm{~h}$

Figure 4: Post treatment extraoral and intraoral photographs

Figure 5: Post treatment intraoral periapical radiograph of 21 
regular follow up to internist and periodontist at every alternate orthodontic appointment.

As the patient was concerned with the unesthetic appearance of the gap between two maxillay central incisors, treatment included only the maxillary arch ignoring mild crowding of lower anteriors. Bondable buccal tubes were used instead of bands taking into account patient's medical condition and risk of periodontal breakdown. 0.022" MBT brackets were bonded on maxillary teeth. The treatment progressed gradually with levelling and alignment of teeth excluding 21. After the levelling and alignment of other maxillary teeth except 21, a rigid 0.018 " stainless steel arch wire was inserted. Then lingual buttons were bonded on the labial and palatal surface of 21 and couple was used to derotate it. (Fig. 3) After the derotation was completed, esthetic contouring was done with composite. (Fig. 4)

Light force was used during all stages of the treatment. Stainless steel ligatures were preferred to elastomeric modules due to less plaque retentive property. Maintenance of adequate oral hygiene was reinforced throughout the treatment period. Fixed lingual retainer with 'flexible spiral wire' was bonded to retain the achieved result after an active treatment duration of 9 months. (Fig. 4) Post treatment intra-oral periapical radiograph showed mild blunting of the root apex of 21 which is acceptable for maxillary incisors during orthodontic treatment. $^{10}$ (Fig. 5) Pericison was further performed after consultation with periodontist regarding periodontal health and internist for glycemic control.

\section{DISCUSSION}

Age is not a restriction for orthodontic treatment. Orthodontic treatments of adult do have some limitations which can be overcome with carefully designed treatment plan involving multidisciplinary approach. ${ }^{11}$ Though initiation of tooth movement takes a longer time, excellent cooperation received from adult patients makes up for the initial slow tooth movement. ${ }^{12}$ Several authors conclude that age do not seem to play a role in the overall duration of the treatment. ${ }^{13}$ However, integrity of tooth and surrounding tissues should be ensured and underlying systemic conditions need to be addressed prior to the initiation of treatment.

With adequate monitoring of patient's glycemic levels and slow, gradual increase in force, orthodontic treatment is possible in patients with diabetes. $^{5}$ The maintenance of oral hygiene is important in avoiding bacterial plaque retention, especially with patient's increased risk of periodontal disease which is a concern for diabetic patients. $^{9}$ The diabetic patients are vulnerable to periodontitis $^{14}$ which is defined as clinical attachment loss of $2 \mathrm{~mm}$ or more. ${ }^{15}$ Bone metabolism is adversely affected by both the direct impact of hyperglycemia and the long-term effects of vascular disease. Furthermore, patients whose diabetes is inadequately controlled tend to show a greater loss alveolar bone than patients with wellcontrolled diabetes.

Orthodontic management in this adult patient with diabetes was further challenged by severe rotation of maxillary left central incisor secondary to mesiodens. Mesiodens often lead to uneruption, ectopic displacement or rotation of maxillary central incisor. ${ }^{16}$ Extraction of mesiodens in early mixed dentition may allow spontaneous eruption or alignment of maxillary central incisor. ${ }^{17}$ But in the present case, the patient approached the orthodontist in his late fifties only after the extraction of painful mesiodens and appearance of unesthetic diastema.

Correction of incisor rotation can be done with removable appliances like acrylic plate incorporating Z-spring, modified removable plate ${ }^{18}$ or whip appliance ${ }^{19,20}$. One point contact with a removable appliance leads to tipping which is not desired. Further the question of compliance always exists with the use of removable appliances. In fixed appliances like $\mathrm{Begg}^{21}$ or Tip-Edge ${ }^{22}$, derotating springs are used but such springs are not common for edgewise or preadjusted straight wire appliances. In this case, couple was used to derotate the affected central incisor after levelling and alignment of adjacent teeth so that a rigid arch wire would preserve the arch form.

\section{CONCLUSION}

Orthodontic treatment can be carried out in diabetic adult patient and age is not a restriction provided 
that the diabetes is under control. However, integrity of the tooth and surrounding tissues should be ensured and measures for maintenance of adequate oral hygiene should be undertaken.

\section{ACKNOWLEGEMENT}

I am thankful to the patient who gave consent for the publication of his photographs in this article.

\section{REFERENCES}

1. Keim RG, Gottlieb EL, Nelson AH, Vogels 3rd DS. 2005 JCO orthodontic practice study. part 1: trends. J Clin Orthod. 2005 Nov;39(11):641-50. PMID:16380656.

2. Nattrass C, Sandy J. Adult orthodontics--a review. British Journal of Orthodontics. 1995;22(4):331-7. DOI:10.1179/ bjo.22.4.331; PMID:8580099.

3. Moshkelgosha V, Azar H, Golkari A, Azar MR. Utilization of orthodontic services in the Fars Province, Iran: the reasons people travel to the capital for orthodontic treatment. Journal of Dentistry. 2015 Sept;16(3):195-9.

4. Proffit WR, Fields Jr HW, Sarver DM. Contemporary orthodontics. $5^{\text {th }}$ ed. St. Louis: Mosby Elsevier Health Sciences; 2014.

5. Bagga DK. Limitations in adult orthodontics: a review. Journal of Oral Health and Community Dentistry 2009;3:52 -5 .

6. Christensen L, Luther F. Adults seeking orthodontic treatment: expectations, periodontal and TMD issues. British Dental Journal. 2015;218(3):111-7.DOI:10.1038/ sj.bdj.2015.46; PMID:25686427.

7. Löe H. Periodontal disease: the sixth complication of diabetes mellitus. Diabetes Care. 1993;16(1):329-34. DOI:10.2337/diacare.16.1.329; PMID:8422804.

8. Krishnan V, Davidovitch Ze. Biological mechanisms of tooth movement. $2^{\text {nd }}$ ed. New Jersey: John Wiley \& Sons; 2015. DOI:10.1002/9781118916148.

9. Pithon MM, Ruellas CV, Ruellas AC. Orthodontic treatment of a patient with type 1 diabetes mellitus. Journal of Clinical Orthodontics. 2005 Jul;39(7):435-9. PMID:16100417.

10. Weltman B, Vig KW, Fields HW, Shanker S, Kaizar EE. Root resorption associated with orthodontic tooth movement: a systematic review. American Journal of Orthodontics and Dentofacial Orthopedics. 2010;137 (4):462-76. DOI:10.1016/j.ajodo.2009.06.021. PMID: 20362905.

11. Kalia S, Melsen B. Interdisciplinary approaches to adult orthodontic care. J Orthod 2001;28: 191-6.

12. Bagga DK. Adult orthodontics versus adolescent orthodontics: an overview. J Oral Health Comm Dent. 2010;4(2):42-7.

13. Mavreas D, Athanasiou AE. Factors affecting the duration of orthodontic treatment: a systematic review. The European Journal of Orthodontics. 2008;30(4):386-95. DOI:10.1093/ejo/cjn018; PMID:18678758.

14. Report of the expert committee on the diagnosis and classification of diabetes mellitus. Diabetes Care. 2003;26 Suppl 1:S5-20. DOI: 10.2337/diacare.26.2007.S5; PMID:12502614.

15. Lalla E, Cheng B, Lal S, Tucker S, Greenberg E, Goland R, et al. Periodontal changes in children and adolescents with diabetes: a case-control study. Diabetes Care. 2006 Feb;29 (2):295-9. DOI:10.2337/diacare.29.02.06.dc05-1355; PMID:16443876.

16. Meighani G, Pakdaman A. Diagnosis and management of supernumerary (mesiodens): a review of the literature. Journal of Dentistry (Tehran, Iran). 2010;7(1):41-9. PMCID: PMC3184724.

17. Russell KA, Folwarczna MA. Mesiodens-diagnosis and management of a common supernumerary tooth. Journal of Canadian Dental Association. 2003;69(6):362-7. PMID:12787472.

18. Sandhu V. Management of Torsiversion of a Tooth Secondary to a Mesiodens. Indian Journal of Dental Education. 2011;4(3-4):61-3.

19. Jahanbin A, Baghaii B, Parisay I. Correction of a severely rotated maxillary central incisor with the Whip device. The Saudi Dental Journal. 2010;22(1):41-4. DOI:10.1016/ j.sdentj.2009.12.003; PMCID:PMC3804962.

20. Parisay I, Boskabady M, Abdollahi M, Sufiani M. Treatment of severe rotations of maxillary central incisors with whip appliance: report of three cases. Dental Research Journal. 2014;11(1):133-9. .

21. Begg PR, Kesling PC. Begg orthodontic theory and technique. $3^{\text {rd }}$ ed. Florida: WB Saunders Company; 1977.

22. Kesling PC. Dynamics of the Tip-Edge bracket. American Journal of Orthodontics and Dentofacial Orthopedics. 1989;96(1):16-25. DOI: 10.1016/0889-5406(89)90224-2. 